\title{
Modelling the Role of Dietary Habits and Eating Behaviours on the Development of Acute Coronary Syndrome or Stroke: Aims, Design, and Validation Properties of a Case-Control Study
}

\author{
Christina-Maria Kastorini, ${ }^{1,2}$ Haralampos J. Milionis, ${ }^{1}$ John A. Goudevenos, ${ }^{1}$ \\ and Demosthenes B. Panagiotakos ${ }^{2}$ \\ ${ }^{1}$ School of Medicine, University of Ioannina, 45110 Ioannina, Greece \\ ${ }^{2}$ Department of Nutrition Science and Dietetics, Harokopio University, 17671 Athens, Greece \\ Correspondence should be addressed to Demosthenes B. Panagiotakos, d.b.panagiotakos@usa.net
}

Received 2 August 2010; Accepted 25 August 2010

Academic Editor: Christina Chrysohoou

Copyright ( $) 2011$ Christina-Maria Kastorini et al. This is an open access article distributed under the Creative Commons Attribution License, which permits unrestricted use, distribution, and reproduction in any medium, provided the original work is properly cited.

In this paper the methodology and procedures of a case-control study that will be developed for assessing the role of dietary habits and eating behaviours on the development of acute coronary syndrome and stroke is presented. Based on statistical power calculations, 1000 participants will be enrolled; of them, 250 will be consecutive patients with a first acute coronary event, 250 consecutive patients with a first ischaemic stroke, and 500 population-based healthy subjects (controls), age and sex matched to the cases. Socio-demographic, clinical, dietary, psychological, and other lifestyle characteristics will be measured. Dietary habits and eating behaviours will be evaluated with a special questionnaire that has been developed for the study.

\section{Introduction}

Cardiovascular disease (CVD) is the leading cause of morbidity and mortality at a global level, with a significant impact on quality of life as well as an important economic burden [1]. In fact, in 2002, it is estimated that 7.2 million people died from coronary heart disease and 5.5 million from stroke, while according to the WHO estimates and due to the demographic changes, the number of CVD events is expected to increase further [2]. Therefore, prevention of CVD is now considered of major public health importance. Means for reducing the burden of the disease at population level include lifestyle interventions, and particularly dietary modifications.

During the last decades, studies from all over the world have evaluated the relationship between specific foods and dietary patterns with the development of CVD, and particularly coronary heart disease [3-8]. Healthy dietary patterns, like the Mediterranean, characterized by high consumption of foods of plant origin, fruits, vegetables, whole grain cereals, legumes, as well as poultry and fish, have been associated with decreased risk of the disease. On the contrary, more western dietary patterns, characterized by increased consumption of red and processed meat, sweets and desserts, potatoes, and refined cereal are associated with increased risk. However, the role of diet on the development of ischaemic stroke is not that well established $[9,10]$. Moreover, although significant scientific evidence exists regarding the role of specific foods and dietary patterns on the development of CVD, the influence of certain dietary behaviours and practices has not been extensively studied and understood. For example, apart from the type of foods that are actually consumed, meal frequency, breakfast consumption, food consumption in parallel with other activities (such as working or television viewing), systematic consumption of heavy meals or eating alone, as well as sleeping patterns may also play an important direct or indirect role, regarding the development of coronary heart disease and stroke [11-15] (Figure 1).

Thus, the aim of the present study is to evaluate the role of dietary habits and eating behaviours on the likelihood 


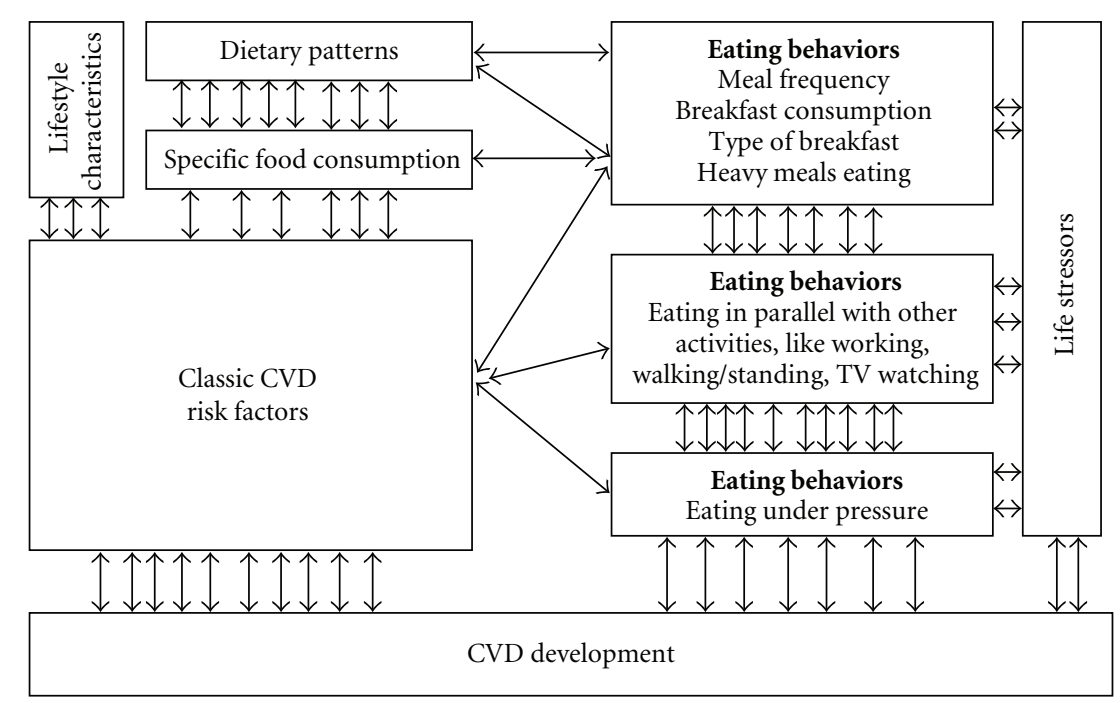

FIGURE 1: A conceptual model about dietary patterns, eating and lifestyle behaviours, and practices that will be tested in this study regarding the development of CVD.

of developing a first CVD event (acute coronary syndrome or stroke), after taking into account other lifestyle and environmental factors as well as socio-demographic and clinical characteristics.

\section{Materials and Methods}

2.1. Design. Multicentre, case-control with individual, age (within \pm 3 years) and sex matching (Figure 2).

2.2. Sampling Procedure. According to the sampling procedure all consecutive patients with a first acute coronary syndrome (ACS) event (acute myocardial infarction (AMI) or unstable angina (UA)) or ischaemic stroke, and without any suspicion of previous CVD, that will enter in the cardiology, pathology clinics, or the emergency units of three major General Hospitals in Greece (i.e., University General Hospital of Ioannina, Korgialeneio-Benakeio Red Cross Hospital, and Alexandra General Hospital, Athens) between October 1, 2009 and December 31, 2010 will be contacted to enrol in the study. Patients with a history of neoplasia or chronic inflammatory disease, as well as individuals with recent changes in their dietary habits, will not be included. Control subjects will be selected on a random, volunteer basis, and they will be without any clinical symptoms or suspicions of CVD in their medical history, as this will be assessed by a cardiologist. The control subjects will be allocated at population basis, and from the same region of the patients. Based on a priori statistical power analysis, a sample size of 500 patients (250 ACS, 250 stroke) and 500 ageand sex-matched healthy subjects, is adequate to evaluate two-sided odds ratios equal to 1.20 , achieving statistical power greater than .80 at .05 probability level $(P$ value).
2.3. Diagnosis of ACS or Stroke. At hospital entry clinical symptoms will be evaluated and a 12-lead electrocardiogram will be performed, by a cardiologist. Evidence of myocardial cell death will be assessed with blood tests and measurement of the levels of troponin I and the MB fraction of total creatinine phosphokinase (CPK). According to the Universal Definition of Myocardial Infarction (Joint ESC/ACCF/AHA/WHF Task Force) [16], blood samples will be obtained on hospital admission, at 6 to $9 \mathrm{~h}$, and again at 12 to $24 \mathrm{~h}$ if earlier samples will be negative and the clinical index of suspicion is high. Acute coronary syndromes, and particularly myocardial infarction (AMI) will be defined by detection of rise and/or fall of troponin I or CPK with at least one value above the 99th percentile of the upper reference limit as well as with at least one of the following features: (a) compatible clinical symptoms, (b) ECG changes indicative of new ischaemia (new ST-T changes or new left bundle branch block LBBB), (c) development of pathological Q waves in the ECG, (d) imaging evidence of new loss of viable myocardium or new regional wall motion abnormality [16]; unstable angina (UA) will be defined by the occurrence of one or more angina episodes, at rest, within the preceding $48 \mathrm{~h}$, corresponding to class III of the Braunwald classification [17]. Ischaemic strokes will be defined through symptoms of neurologic dysfunction of acute onset of any severity, consistent with focal brain ischaemia and imaging/laboratory confirmation of an acute vascular ischaemic pathology [18].

2.4. Anthropometric Characteristics. Body weight (in kilograms) and height (in meters) will be measured following standard procedures (i.e., height will be measured to the nearest $0.5 \mathrm{~cm}$, without shoes, back square against the wall tape, eyes looking straight ahead, while weight will be measured with a lever balance, to the nearest $100 \mathrm{~g}$, without shoes, in light undergarments). Due to possible difficulties 


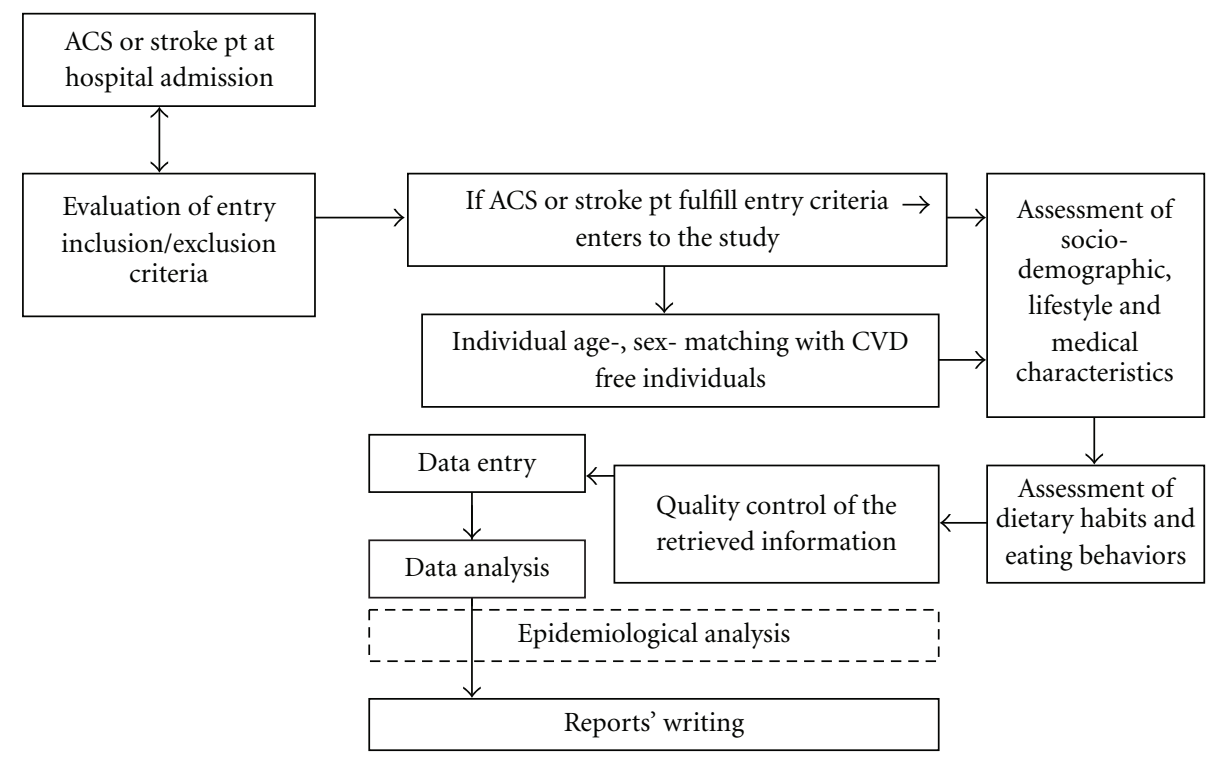

FIGURE 2: Flowchart of the study.

in the assessment of these anthropometric characteristics for the patients, it will be recorded whether the above values are self-reported or measured. Body mass index will then be calculated as weight (in kilograms) divided by standing height (in meters squared) and overweight and obesity will be defined as body mass index $25.0-29.9 \mathrm{~kg} / \mathrm{m}^{2}$ and $>29.9 \mathrm{~kg} / \mathrm{m}^{2}$, respectively. Additionally participants will be asked what their lower and higher body weight was after the age of 20 years (in kilograms). Moreover, the participants will be asked if they have gained or lost weight during the last three months. In case they did, the kilograms gained or lost, and if the gain or the loss were voluntary or not will be recorded.

2.5. Socio-Demographic Characteristics. Socio-demographic variables that will be recorded are age and sex (for the matching procedure), educational level measured by years of school, type of occupation (in the following categories: civil servant, private employee, part-time employee, freelancer, rentier, retired, unemployed, housewife) and occupational skills that will be evaluated through a nine-point scale (values 1-3 refer to manual labour, while values from 7 to 9 refer to intellectual labour). Marital status categorised as single, married, divorced, or widowed and number of children will also be recorded. Financial status will be indirectly evaluated using (a) an index measuring how satisfied the participant is from his/her income (i.e., value 1 means not at all satisfied, to value 9 which means very satisfied), (b) the number of cars in the family, (c) the number of rooms in the house (including kitchen and bathroom), and (d) whether the residence is owned or not.

2.6. Lifestyle Characteristics. Physical activity will be assessed using the International Physical Activity Questionnaire (IPAQ) index [19] that has been validated for the Greek population, too [20]. Subjects will be asked to recall the number of days and hours or minutes they engaged in physical activity of different intensities for at least ten minutes, vigorous intensity and moderate intensity, walking and time spent sitting. According to their physical activity levels, participants will be classified as inactive, minimally active, or health enhancing physical activity (HEPA) active.

Furthermore, sleeping patterns will be assessed and participants will be asked about the hours they sleep at night, if they nap during the day (almost never, only on holidays, sometimes per week, almost every day), and in case they do, for how long (in minutes). They will also be asked about the frequency of night shifts at work (less than once in three months, 1-3 times per month, 2-4 times per week, almost every day). Television viewing will also be assessed by the hours of television viewed daily (less than $1 \mathrm{~h}, 1-2 \mathrm{~h}, 3-5 \mathrm{~h}$, more than $5 \mathrm{~h}$ ) and frequency of food consumption in front of the TV will be recorded (less than once in three months, 1-3 times per month, 2-4 times per week, almost every day).

Current or former smoking habits will be recorded and participants will be classified as (a) current smokers, (b) former smokers, or (c) non-smokers. Particularly, current smokers will be defined as those who smoke at least one cigarette per day, former smokers as those who had stopped smoking more than one year previously, and the rest of the participants will be defined as non-current smokers. Additionally, current and former smokers will be asked about the age they started smoking, the total number of years they smoke, and the number of cigarettes they smoke/smoked daily. Former smokers will be also asked about the number of years they have stopped smoking. Moreover, current and former smokers will be asked about the type of smoke they prefer/preferred (i.e., regular cigarettes, light cigarettes, tobacco), whether they smoke/smoked pipe or cigars, and if they smoke/smoked in their workplace, at home, or in front of their children. For the evaluation of passive smoking, 
participants will be asked if other colleagues smoke in front of them in the workplace for more than $30 \mathrm{~min}$ daily and if other people in their environment smoke in front of them for more than $30 \mathrm{~min}$ per day (partner, parents, children, roommates). If they are not currently exposed to passive smoking, participants will be asked if they were exposed in passive smoking in the past and the years of exposure to passive smoking will be recorded.

2.7. Assessment of Dietary Habits. Dietary habits of the past year will be assessed through a 90-item, validated semi-quantitative food-frequency questionnaire (FFQ). Its validation properties will be briefly presented below. Regarding the dietary assessment, the participant will be asked how often (i.e., less than 3 months, 1-2 times/3 months, 1-2 times/month, 2-4 times/month, 1-2 times/week, 3-5 times/week, almost every day, more than one time per day) he/she consumes the following foods and beverages: red meat, processed meat, poultry, fish (and more specifically baked/boiled fish, fried fish, fresh tuna, or swordfish), legumes, cooked vegetables, pasta and rice (and in particular white pasta, whole wheat pasta, white rice, brown rice), potatoes, salads and fresh vegetables (and more specifically green leafy, cruciferous, coloured or starchy vegetables), eggs, sweets (and in particular baked sweets, honey, marmalade, cakes, white or milk chocolate, dark chocolate), consumption of non-homemade food and type of food (fast-food, sandwich, restaurant), salted nuts, unsalted nuts, canned food, milk and yogurt (and in particular full fat, low fat or skim), the number of milk and yogurt servings consumed in one week, feta-cheese, low fat white cheese, yellow cheese, low fat yellow cheese, and the number of servings of cheese consumed in one week. Fruit consumption will be recorded in fruits per day. The frequency of consumption (rarely, monthly, weekly, daily) of the following sources of fat will also be evaluated: olive oil for cooking or salad dressing, olive oil for frying, seed oil for cooking or salad dressing, seed oil for frying, mayonnaise or other sauce, butter, margarine, milk cream, olives. Additionally, the type of olive oil (packed extra-virgin olive oil, packed virgin olive oil, packed refined olive oil, unpacked olive oil-production by the participant, unpacked olive oil bought from friends) and the weekly amount (in litters) consumed will be assessed. Furthermore, bread as well as rusk consumption will be evaluated according to slices of bread consumed daily (less than half a slice, 0-1, 1-2, 3-4, 5-6, over 7 slices) and according to rusks consumed daily (less than one, 1-2, 3-4, $5-6,7-8$, over 9 ). The type (white or whole wheat) of bread and rusk consumption will be also recorded (frequency: rarely, monthly, weekly, and daily). Salt consumption in cooking, and the use of table salt or salt substitute will also be assessed. In addition, water, beverages, and juice consumption will be assessed in glasses per day. The type of beverage (cola drink, soda, light) and juice (carbonated, noncarbonated, from fresh fruits) will be evaluated. Frequency of alcohol consumption will be assessed in four categories: rarely, monthly, weekly, and daily. The participant will be also asked if he/she consumed more alcohol in the past as compared with the present consumption. Furthermore, the type of drink consumed will be recorded (i.e., beer, white wine, red wine, whisky, vodka, or ouzo) and the amount of alcohol consumed will be measured in wineglasses per day (i.e., <1, 1-2, 3-4, 5-6, over 7 wineglasses; each wineglass will be equivalent to $12 \mathrm{~g}$ of ethanol). Additionally, alcohol consumption patterns will be recorded; in particular, drinking of large quantities of alcohol rarely (for example in the weekends) as well as the frequency of hangover during the last year (i.e., never, 1-2 times/year, 1-2 times/ 6 months, 1-2 times/month, over twice/month). Coffee consumption will be assessed regarding its frequency (i.e., rarely, monthly, weekly, and daily). The participants will be asked about the type of coffee they prefer to drink (Greek, instant coffee, filtered, espresso, decaffeinated, cappuccino) as well as about the cups of coffee consumed daily $(<1,1-2,3-4$, 5-6, over 7). Additionally, the amount of coffee added in teaspoons in each cup, the type of coffee consumed (light, medium, heavy), and whether sugar or other sweetener is added and if yes, how many teaspoons are added will be evaluated. Frequency (i.e., rarely, monthly, weekly, daily) of tea consumption, the number of cups of tea per day (i.e., $0-1,1-2,3-5$, over 5 cups) as well as the type of tea consumed (mountain tea, green tea, black tea, chamomile tea) will be also assessed. Additionally, participants will be asked whether they add sugar or other sweetener, and if yes how many teaspoons they add. Furthermore, the participants will be asked if they are on a diet and if they have changed their dietary patterns during the last year. Participants with important recent (i.e., within a year) changes in their dietary habits will be excluded from the study.

Overall assessment of dietary habits will be performed using the MedDietScore [21], an eleven-item composite index that evaluates adherence to the Mediterranean dietary pattern. The range of the MedDietScore is between 0-55. Higher values of this diet score indicate greater adherence to the Mediterranean diet. The validation properties of the MedDietScore have been presented elsewhere in the literature [21-23].

2.8. Validation of the FFQ. As mentioned above, a semiquantitative FFQ developed for the purposes of this study will be used. For the validation properties, 59 males (40 \pm $14 \mathrm{yrs})$ and 77 females ( $40 \pm 13$ yrs) were asked to complete the FFQ presented above, during 2010. Participants were also asked to complete a 3-day food record of what they have eaten. The recording period included two weekdays and one weekend day, over the same time span as the FFQ. This record was used as the reference method for validating the FFQ. Agreement of the FFQ with the 3-day food records was evaluated using the Bland-Altman method and the Kendall's tau-b coefficient. Between the 3-day food records and the FFQ, moderate agreement for coffee (tau-b $=0.56, P<$ .001 ), fruits (tau-b $=0.48, P<.001$ ), fast-food consumption (tau-b $=0.47, P<.001)$, alcohol (tau-b $=0.47, P<.001)$, sweets (tau-b $=0.36, P<.001)$, vegetables (tau-b $=0.32$, $P<.001$ ), and red meat (tau-b $=0.31, P<.001$ ) was found, while low, but still significant agreement for greens 
(tau-b $=0.22, P=.004)$, cereals (tau-b $=0.21, P<$ .001 ), and dairy products (tau-b $=0.18, P=.007$ ) was observed. According to the Bland-Altman method the level of agreement varied from $90 \%$ to $99 \%$. Sensitivity analyses by sex and age category ( $<$ or $>55$ yrs) and obesity status showed similar validity of the FFQ in each subgroup.

2.9. Assessment of Eating Behaviours. As mentioned in the aim of the study, a research hypothesis that will be also tested is whether certain eating behaviours may influence the likelihood of developing ACS or stroke. Thus, a special questionnaire that has been designed for the purposes of the present study will be used to evaluate several behaviours of the participants such as meal frequency, breakfast consumption, consumption of food in parallel with other activities. Frequency of consumption (rarely, 1-2 times/week, 3-5 times/week, and almost every day) of the following meals and snacks will be assessed: breakfast, morning snack, lunch, evening snack, dinner, and bed-time snack. Consumption of any food except water will be considered as a meal or snack. Additionally, potential reasons for skipping a meal will be evaluated. Participants will be asked about how frequently (less than once in three months, 1-3 times per month, 24 times per week, almost every day) they skip a meal or snack, because of hard work, because of the will to lose weight, or because they are not hungry. Detailed information will be asked regarding breakfast consumption. In particular, participants will be asked about the time they eat breakfast (earlier than $6 \mathrm{am}, 6-8 \mathrm{am}, 8-10 \mathrm{am}$, after $10 \mathrm{am}$ ) and the frequency (rarely, 1-2 times/week, 3-5 times/week, almost every day) they consume the following foods for breakfast: coffee or tea without sugar, coffee or tea with sugar, milk and yogurt, juice, fruits, cereals and rusks, sandwiches, bread, marmalade, honey, bakery products (croissants, cakes etc.), eggs, omelettes, and processed meat. Moreover, duration of lunch and dinner (i.e., $0-15 \mathrm{~min}, 15-30 \mathrm{~min}, 30-45 \mathrm{~min}$, 45-60 $\mathrm{min}$, and over $60 \mathrm{~min}$ ), consumption of alcohol with meals (i.e., no alcohol consumption, red wine, white wine, beer, and other) and the time (in minutes) between dinner and night sleep will be recorded.

Additionally, frequency (rarely, 1-2 times per week, 35 times per week, almost every day) of food consumption under stress conditions (before the participant has time to relax), while working at the same time (without being on a break), and while walking or standing (not sitting) will be recorded. Furthermore, participants will be asked about how often they consume a more heavy meal that makes them feel full (less than once in three months, 1-3 times per month, 2-4 times per week, almost every day), if they are responsible for the preparation of meals (almost never, sometimes per week, a meal of the day, almost every meal), and how frequently they eat alone (almost never, sometimes per week, a meal of the day, almost every meal).

The last meal consumed before the ACS or the stroke event will be recorded (breakfast, morning snack, lunch, evening snack, dinner, and bed-time snack) and patients will be asked if they have consumed more food than usual the day of the event or the previous day or more heavy food than usually, and which type of food. They will also be asked if they have consumed more alcohol than usually (and how many wineglasses) and if they have consumed more coffee (and how many cups) in the day of the event or the previous day. Time between the last meal consumed and the event will be also recorded. Patients will be asked if they were feeling full or hungry at the time of the event, using a 9-item scale (1: very full, 9: very hungry). Furthermore, they will be asked if the day of the event or the previous day they were feeling: angry or scared, depressed or stressed, if they had exercised more than usual, if they had not slept at night, if they were ill, and if they were exposed to cold. Controls will be asked the same questions, but regarding the day of the interview or the previous day.

To evaluate the participants' health perspectives they will be asked to value the importance of several CVD risk factors using a scale of 1 to 9 ( 1 means not at all important whereas 9 means very important). The factors that will be evaluated are smoking, passive smoking, sedentary lifestyle, stress, unhealthy dietary habits, overweight and obesity, diabetes, hypercholesterolemia or hypertension, family history.

2.10. Assessment of Medical History. In all participants, family history of CVD as well as personal and family history of hypertension, hypercholesterolemia, hypertriglyceridemia, and diabetes will be recorded. In case of positive responses regarding personal history of the above conditions, the participant will be asked about the way of management (diet and/or drugs) and the frequency of drug use (daily, weekly, monthly, rarely) in case they do not adhere to the drug prescription. Participants will be also asked if they have renal failure (and if yes for how many years), peripheral artery disease and thyroid disease (hypothyroidism, hyperthyroidism, way of management, years of thyroid disease). Women will be asked about their menopause status (premenopausal, menopausal less than 2 years, or menopausal more than 2 years) and also about potential hormone use (oral contraceptive pills, menopause hormone replacement therapy) and for how many years. Finally, angiographic data and the following clinical and biochemical values will be recorded from the latest participants' record: blood pressure, heart rate, fasting glucose, total cholesterol, LDL-cholesterol, HDL-cholesterol, triglycerides, TSH, hematocrit, white blood cells count, platelets count, urea, creatinine, and uric acid.

2.11. Psychological Evaluation. A previously translated and validated version of the Zung Depression Rating Scale (ZDRS) will be used for the assessment of depressive symptoms [24, 25]. The ZDRS is a self-rating scale consisting of 20 items that cover affective, psychological, and somatic symptoms for the measurement of depression, and was originally developed in order to assess depression symptoms without the bias of an administrator affecting the results. The individual specifies the frequency a symptom is experienced (i.e., little $=1$, some $=2$, a good part of the time $=3$, or most of the time $=4$ ). Total theoretical range of the score is $20-80$, with higher scores indicating more severe depression 
[24]. Scores 20-49 are considered normal, scores of 5059 indicate mild depression, scores of 60-69 moderate to marked depression, while scores of 70-80 severe depression [25].

Moreover, the State-Trait Anxiety Inventory form $\mathrm{Y}$ is a brief self-rating scale for the assessment of state and trait anxiety. In the present study anxiety will be assessed only with the also previously translated and validated version of Spielberger Trait Anxiety Inventory (STAI form Y-2), which is a 20 -item self-reported questionnaire evaluating how the respondent feels generally $[26,27]$. The 20 items are rated from 1 to 4 according to frequency of their feelings (i.e., almost never, sometimes, often, almost always). Total theoretical range of the score ranges from 20 to 80 .

2.12. Bioethics. The study has been approved by the Ethics Committee of the University Hospital of Ioannina and will be carried out in accordance to the Declaration of Helsinki (1989) of the World Medical Association. Prior to the collection of any information, participants will be informed about the aims and procedures of the study and will provide their written signed consent.

2.13. Statistical Analysis Plan. Normally distributed continuous variables will be presented as mean values \pm standard deviation, skewed variables as median and quartiles and categorical variables as frequencies. Associations between categorical variables will be tested by the calculation of chi-squared test. Comparisons between normally distributed continuous variables will be performed by the calculation of Student's $t$-test. In case of skewed continuous variables, the tested hypotheses will be evaluated using the nonparametric $U$-test suggested by Mann and Whitney. Correlations between continuous variables will be evaluated using the Pearson's $r$ or Spearman rho coefficients. Normality of the variables will be tested using P-P plots. Estimations of the relative probabilities of developing CVD (ACS, stroke or combined) will be performed by the calculation of the odds ratio and the corresponding 95\% confidence intervals through multiple logistic regression analysis. HosmerLemeshow statistic will be calculated to test goodness-of-fit. All reported $P$ values will be based on two-sided tests. SPSS 18.0 software (SPSS Inc., Chicago, Il, USA) will be used for all the statistical calculations.

\section{Study's Expectations}

The findings of this case-control study will provide novel information and valuable explanations and answers on how dietary choices, from specific food consumption to eating behaviours and practices, influence the development of ACS and ischaemic strokes. Prevention of CVD is of considerable public health importance as it constitutes a major public health problem, especially in westernised world, and less than $50 \%$ of its variation has been explained by the up-todate known risk factors. Lifestyle characteristics, like diet, smoking, and physical activity are considered to play a crucial role for the prevention of the disease, because they can be modified. However, in spite of the nutritional guidelines and recommendations for a healthy diet and lifestyle, dietary habits in the developed world, in developing countries at "nutrition transition", and even around the Mediterranean basin are changing towards the opposite direction [28, 29]. Thus, understanding of the role of dietary habits and eating behaviours on the development of CVD could offer other means to focus on prevention through emphasis on these factors. The results of the present study may suggest other possible ways to emphasize when targeting on the prevention of CVD, giving attention not only on types of food consumed and dietary guidelines, but also on eating behaviours, like meal frequency, breakfast consumption, heavy meal consumption, eating alone, activities to be followed or not while consuming a meal, like working, walking, or television viewing. Finally, attention might be needed regarding other lifestyle behaviours like hours of night sleep and napping, as they could also have an effect on the development of cardiovascular disease directly or indirectly (meaning that they could influence as well food choices).

\section{Authors' Contribution}

C. M. Kastorini is the principal investigator of the study and wrote the paper, H. Milionis, J. Goudevenos contributed to the design of the study, and reviewed the paper and D. B. Panagiotakos had the concept, designed the study, and reviewed the paper.

\section{Acknowledgments}

The authors would like to thank the Directors from the Department of Cardiology, Athens Red Cross Hospital and Acute Stroke Unit, Department of Clinical Therapeutics, Alexandra Hospital, University of Athens, Vasileios Nikolaou and Konstantinos N. Vemmos, for their substantial support, as well as to present and thank the field investigators of the study: Aggeliki Ioannidi, Ekavi Georgousopoulou, Eva Ntziou, Markella Symeopoulou, Zoe Konidari, Kallirroi Kalantzi, Vaia Salma, Dimitrios Kantas, Eftychia Bika, Michael Kostapanos, Antonis Kramvis, Glykeria Papagiannopoulou, Alexandra Litsardopoulou, Eirini Trichia, Dimitris Potsios, Vassiliki Vlachaki, Fani Lioliou, Labros Papadimitriou, Konstantina Siganou, and Ioanna Kousoula. C. M. Kastorini received grants to perform this study from the National Scholarships Foundation and the Hellenic Atherosclerosis Society.

\section{References}

[1] AHA. American Heart Association, Heart and Stroke Statistical Update, American Heart Association, Dallas, Tex, USA, 2008.

[2] World Health Organization, "The top 10 causes of death," March 2009, http://www.who.int/mediacentre/factsheets/ fs310/en/index.html.

[3] T. T. Fung, K. M. Rexrode, C. S. Mantzoros, J. E. Manson, W. C. Willett, and F. B. Hu, "Mediterranean diet and incidence of and mortality from coronary heart disease and stroke in women," Circulation, vol. 119, no. 8, pp. 1093-1100, 2009. 
[4] S. Lee, L. Harnack, D. R. Jacobs Jr., L. M. Steffen, R. V. Luepker, and D. K. Arnett, "Trends in diet quality for coronary heart disease prevention between 1980-1982 and 2000-2002: the Minnesota Heart Survey," Journal of the American Dietetic Association, vol. 107, no. 2, pp. 213-222, 2007.

[5] A. Keys, A. Menotti, C. Aravanis, et al., "The seven countries study: 2,289 deaths in 15 years," Preventive Medicine, vol. 13, no. 2, pp. 141-154, 1984.

[6] M. de Lorgeril, P. Salen, J.-L. Martin, I. Monjaud, J. Delaye, and N. Mamelle, "Mediterranean diet, traditional risk factors, and the rate of cardiovascular complications after myocardial infarction: final report of the Lyon Diet Heart Study," Circulation, vol. 99, no. 6, pp. 779-785, 1999.

[7] F. B. Hu and W. C. Willett, "Optimal diets for prevention of coronary heart disease," Journal of the American Medical Association, vol. 288, no. 20, pp. 2569-2578, 2002.

[8] J. S. Skinner, C. J. Albers, J. Goudevenos et al., "Prospective study of patients aged 55 years or less with acute myocardial infarction between 1981 and 1985: outcome 7 years and beyond," British Heart Journal, vol. 74, no. 6, pp. 604-610, 1995.

[9] T. O. Cheng, "Influence of dietary patterns on stroke risk in China," Stroke, vol. 36, no. 2, p. 228, 2005.

[10] T. T. Fung, M. J. Stampfer, J. E. Manson, K. M. Rexrode, W. C. Willett, and F. B. Hu, "Prospective study of major dietary patterns and stroke risk in women," Stroke, vol. 35, no. 9, pp. 2014-2019, 2004.

[11] N. Lipovetzky, H. Hod, A. Roth, Y. Kishon, S. Sclarovsky, and M. S. Green, "Heavy meals as a trigger for a first event of the acute coronary syndrome: a case-crossover study," Israel Medical Association Journal, vol. 6, no. 12, pp. 728-731, 2004.

[12] F. Kronenberg, M. A. Pereira, M. K. Schmitz et al., "Influence of leisure time physical activity and television watching on atherosclerosis risk factors in the NHLBI Family Heart Study," Atherosclerosis, vol. 153, no. 2, pp. 433-443, 2000.

[13] H. Campos and X. Siles, "Siesta and the risk of coronary heart disease: results from a population-based, case-control study in Costa Rica," International Journal of Epidemiology, vol. 29, no. 3, pp. 429-437, 2000.

[14] A. Naska, E. Oikonomou, A. Trichopoulou, T. Psaltopoulou, and D. Trichopoulos, "Siesta in healthy adults and coronary mortality in the general population," Archives of Internal Medicine, vol. 167, no. 3, pp. 296-301, 2007.

[15] A. I. Qureshi, W. H. Giles, J. B. Croft, and D. L. Bliwise, "Habitual sleep patterns and risk for stroke and coronary heart disease: a 10-year follow-up from NHANES I," Neurology, vol. 48, no. 4, pp. 904-911, 1997.

[16] K. Thygesen, J. S. Alpert, and H. D. White, "Universal definition of myocardial infarction," Journal of the American College of Cardiology, vol. 50, no. 22, pp. 2173-2195, 2007.

[17] E. Braunwald, Heart Disease, W.B. Saunders, London, UK, 5th edition, 1997.

[18] C. S. Kidwell and S. Warach, "Acute ischemic cerebrovascular syndrome: diagnostic criteria," Stroke, vol. 34, no. 12, pp. 2995-2998, 2003.

[19] C. L. Craig, A. L. Marshall, M. Sjöström et al., "International physical activity questionnaire: 12-country reliability and validity," Medicine and Science in Sports and Exercise, vol. 35, no. 8, pp. 1381-1395, 2003.

[20] G. Papathanasiou, G. Georgoudis, M. Papandreou et al., "Reliability measures of the short International Physical Activity Questionnaire (IPAQ) in Greek young adults," Hellenic Journal of Cardiology, vol. 50, no. 4, pp. 283-294, 2009.
[21] D. B. Panagiotakos, C. Pitsavos, and C. Stefanadis, "Dietary patterns: a Mediterranean diet score and its relation to clinical and biological markers of cardiovascular disease risk," Nutrition, Metabolism and Cardiovascular Diseases, vol. 16, no. 8, pp. 559-568, 2006.

[22] D. B. Panagiotakos, C. Pitsavos, F. Arvaniti, and C. Stefanadis, "Adherence to the Mediterranean food pattern predicts the prevalence of hypertension, hypercholesterolemia, diabetes and obesity, among healthy adults; the accuracy of the MedDietScore," Preventive Medicine, vol. 44, no. 4, pp. 335340, 2007.

[23] D. Panagiotakos, N. Kalogeropoulos, C. Pitsavos, et al., "Validation of the MedDietScore via the determination of plasma fatty acids," International Journal of Food Sciences and Nutrition, vol. 60, supplement 5, pp. 168-180, 2009.

[24] W. W. Zung, "A self-rating depression scale," Archives of General Psychiatry, vol. 12, pp. 63-70, 1965.

[25] K. Fountoulakis, A. Iacovides, S. Samolis et al., "Reliability, validity and psychometric properties of the Greek translation of the Zung Depression Rating Scale," BMC Psychiatry, vol. 1, Article ID 6, 2001.

[26] Spielberger, CDGRLLRE: Manual for the State-Trait Anxiety Inventory (Self-Evaluation Questionnaire), Consulting Psychologists Press, Palo Alto, Calif, USA, 1970.

[27] K. N. Fountoulakis, M. Papadopoulou, S. Kleanthous et al., "Reliability and psychometric properties of the Greek translation of the State-Trait Anxiety Inventory form Y: preliminary data," Annals of General Psychiatry, vol. 5, article 2, 2006.

[28] D. Lairon, "Intervention studies on Mediterranean diet and cardiovascular risk," Molecular Nutrition and Food Research, vol. 51, no. 10, pp. 1209-1214, 2007.

[29] F. Sofi, G. Innocenti, C. Dini et al., "Low adherence of a clinically healthy Italian population to nutritional recommendations for primary prevention of chronic diseases," Nutrition, Metabolism and Cardiovascular Diseases, vol. 16, no. 6, pp. 436-444, 2006. 


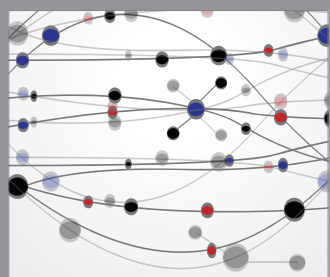

The Scientific World Journal
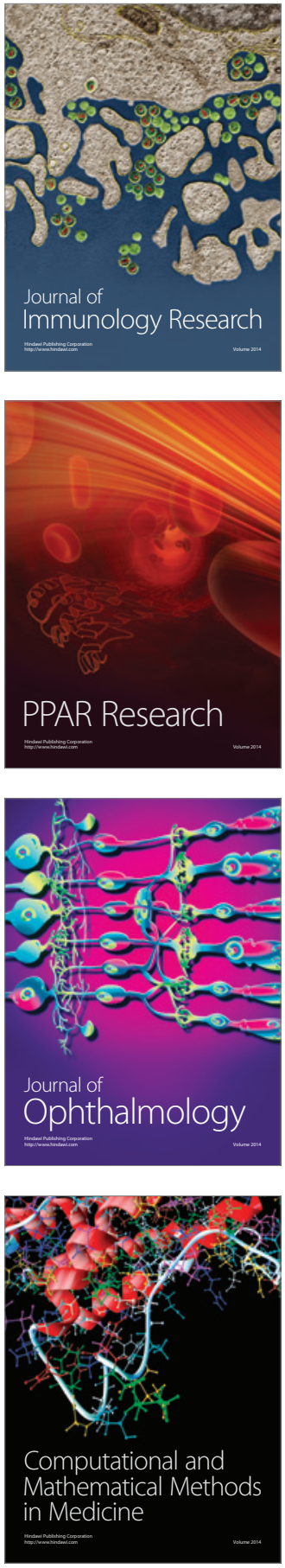

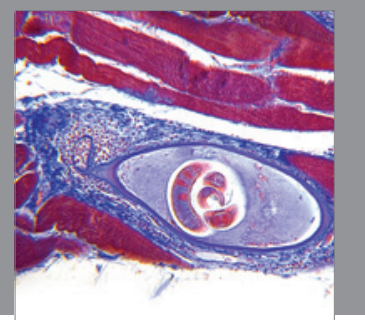

Gastroenterology

Research and Practice
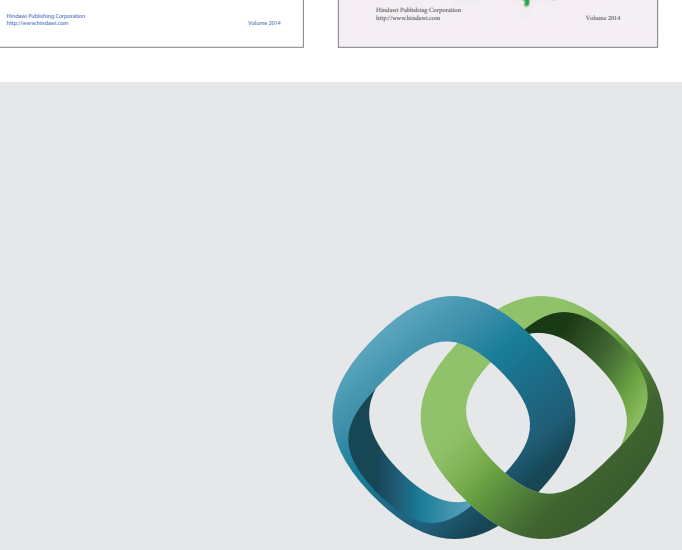

\section{Hindawi}

Submit your manuscripts at

http://www.hindawi.com
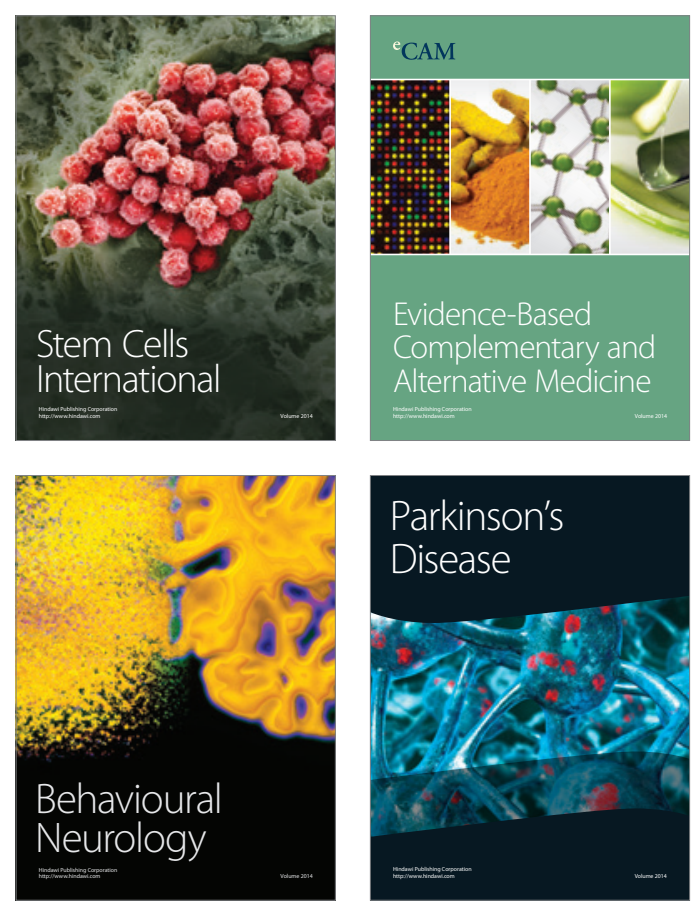

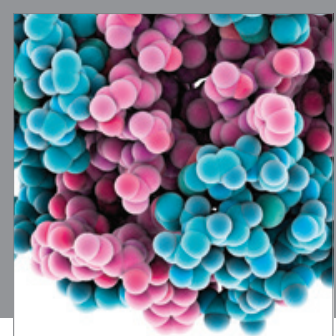

Journal of
Diabetes Research

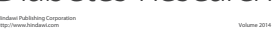

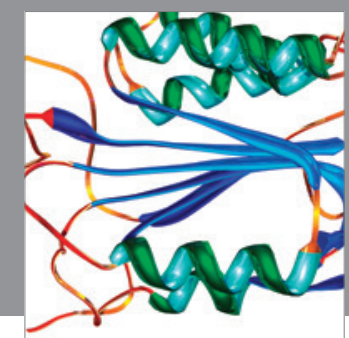

Disease Markers
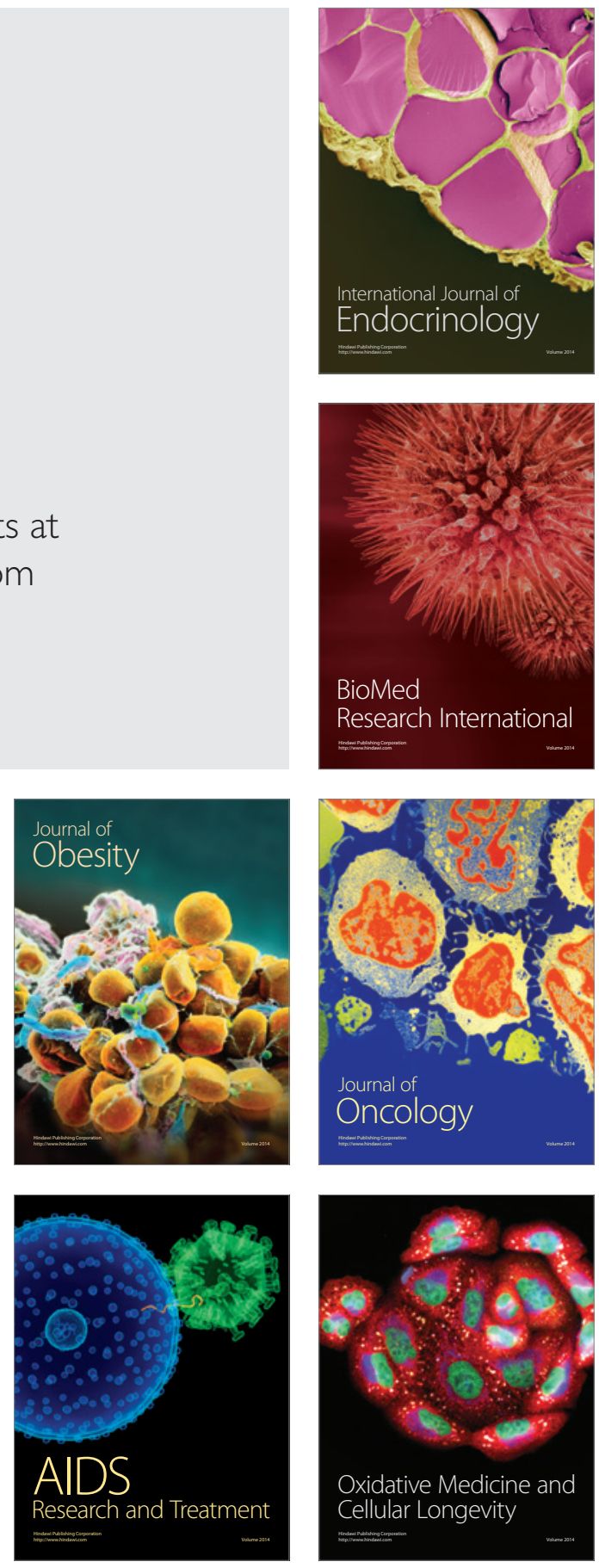